\title{
Proposta para uma visão clínica das lentes progressivas*
}

\section{Purpose to a clinical view of the progressive addition lenses}

Fernando Queiroz Monte', Cleanto Jales de Carvalho Filho'

\section{Resumo}

Objetivos: Estudar as lentes progressivas, à luz da óptica fisiológica, para mostrar os achados obtidos no lensômetro e a sua aplicabilidade na prática diária. Métodos: Depois de explicar a forma de examinar as lentes, foram indicadas as bases físicas que permitiram o estudo, sendo levados em conta as variações do sistema prismático das lentes convexas e côncavas, as diferenças na extensão da zona de progressão e a razão da sua posição diagonal. O estudo foi realizado com 13 pares de lentes de uma mesma empresa e cobrindo todos os desenhos de lentes que ela disponibilizava na época do estudo. Todas as lentes tinham $2 \mathrm{D}$ para longe (uma lente côncava e outra convexa) e as suas adições eram de $2 \mathrm{D}$. Resultados: As lentes progressivas convexas têm na sua correção para longe um prisma de base inferior que depois de apresentar um local sem prisma passa a exibir um prisma de base superior. As lentes côncavas têm prisma de base inferior crescente. Ambas sofrem um desvio vertical médio, na amostra, de 5,86 nas lentes convexas, e $6,93^{\circ}$ nas lentes côncavas. Conclusão: Os autores, baseados nos achados, mostram os fatores que devem ser levados em conta no exame das lentes progressivas para minimizar a insatisfação ao uso dessas lentes. Nos estudos das lentes progressivas é considerado como primeiro plano a progressão sobre o plano secundário dos valores dióptricos enquanto nesse trabalho é considerado principal o valor dióptrico e a progressão secundária.

Descritores: Lentes; Acuidade visual; Satisfação do paciente; Estudo comparativo; Modelos teóricos; Desenho de equipamento

\footnotetext{
'Preceptor de Residência Médica em Oftalmologia do Hospital Geral de Fortaleza e da Sociedade de Assistência aos Cegos - Fortaleza (CE), Brasil.

* Trabalho vencedor do Prêmio Incentivo à Pesquisa Científica do $36^{\circ}$ Concurso Varilux.
} 


\section{INTRODUÇÃO}

A s lentes progressivas ainda hoje são mal compreendidas. Existem opiniões ingênuas como as de Jalie que acha que elas se devem ao aumento do poder dióptrico obtido pelo decréscimo gradual do raio de curvatura da superfície anterior entre as zonas de visão de longe para a de perto ${ }^{(1)}$.

Existem concepções confusas como as de Reiner que diz ser constituída por uma zona elíptica seguida por uma superfície esférica e termina por uma hipérbole numa curva de secção cônica (Kegelschnitte Kurven) e existem outras lentes cuja zona de progressão correria numa linha umbilicada (Nabel-Linie) ${ }^{(2)}$. Esqueceu, no primeiro caso, que para completar o cone precisaria ter uma curva de parábola.

Já Benjamin e Gordon descrevem como a progressão de uma secção cônica de esfericidade mutante a partir de uma elipse inicial achatada nas pontas (oblate ellipsoid), uma superfície esférica, seguida de um elipsóide que se prolonga (prolate ellipsoid) por curvas parabolóide e hiperbolóides ${ }^{(3)}$.

Essa concepção, apesar de ser atual, reproduz o que mostra o trabalho de Arbenz, de 1975, ao que parece, seguindo o que Maitenaz escreveu em 1967. No trabalho de Arbenz, ele coloca a mesma série: Elipse, esférico, elipse, hipérbole e parábola. Esse autor diferencia as elipses, sendo a primeira com o maior diâmetro vertical e a segunda com maior diâmetro horizontal ${ }^{(4)}$.

Em um estudo com cortes do cone em computador, foi mostrado que para explicar as curvas geométricas dos raios nas lentes progressivas, elas deveriam ser superfícies cônicas e, assim sendo, as elipses não seriam diferentes antes e depois do círculo, mas com diâmetros proporcionais e com o maior na posição vertical ${ }^{(5)}$.

Achamos que mesmo esses autores deixaram em aberto como seria adaptado um cone sobre uma lente esférica ou cilíndrica.

As lentes progressivas são vistas atualmente apenas do ponto de vista tecnológico. Os mais aprofundados estudos sobre elas foram feitos por físicos e por optometristas e o empenho feito pelos fabricantes das lentes progressivas, desde que elas foram lançadas, foi para superar os inevitáveis "astigmatismos indesejados" (unwanted astigmatism).

Os oftalmologistas não tiveram interesse de conhecer melhor estas lentes do ponto de vista da óptica fisiológica, portanto do emprego na clínica. Ao nível dos optometristas, é chamado de exame clínico a indagação do grau de satisfação demonstrado pelo usuário das len- tes e ao analisá-la levam em conta as marcações formais que vêm nas lentes. Baseiam-se apenas na subjetividade $^{(6.8)}$. Já houve estudo, e identificamos um isolado e, pelo que sabemos, sem seqüência mesmo pelo autor, que procurou situar dentro da área de atenção do médico e partindo das qualidades ópticas das lentes progressivas. ${ }^{(9)}$.

\section{Métodos}

O material usado foram produtos da empresa SOLA $^{\mathrm{TM}}$, de quem obtivemos 13 pares de blocos com Adição de Esf +2,00D. A surfaçagem das lentes foi feita por um único laboratório seguindo as recomendações do fabricante e os exames das lentes foram procedidos num Lensômetro Topcon computadorizado CL2500 ${ }^{\oplus}$.

\section{Método de exame das lentes}

As lentes progressivas foram examinadas com focos de luz do lensômetro graduados tanto para $0,5 \mathrm{~mm}$ como para $0,7 \mathrm{~mm}$, dependendo do direcionamento da pesquisa. As unidades usadas foram $0,25 \mathrm{D}$ e $0,01 \mathrm{D}$.

Para o exame das lentes adotamos o seguinte método:

1 - Utilizando o foco de $7 \mathrm{~mm}$ ou de $5 \mathrm{~mm}$, de acordo com o dado pesquisado, colocávamos o foco do lensômetro na parte superior da lente e ajustávamos o lensômetro para o valor dióptrico encontrado naquela altura (Figura $1 \mathrm{~A}$ ).

2 - Descíamos o foco do lensômetro, ou subíamos a lente, pela parte em que não havia aberração astigmática, com os valores do ajuste feito na parte superior, até o ponto em que começava a haver aumento do poder dióptrico da lente. Tomávamos como representando o centro óptico para longe o ponto mais baixo que tivesse o mesmo valor dióptrico do encontrado na parte superior (Figura 1B).

3 - O ponto vl era marcado, anotávamos o valor dióptrico e o valor prismático correspondente desde que o tomávamos como o centro óptico de longe por-
A

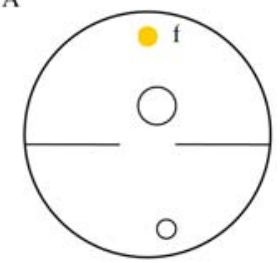

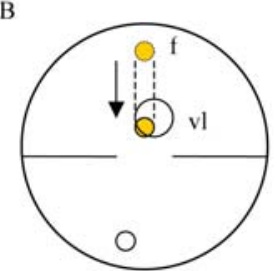

Figura 1: No lado esquerdo (1A) o foco do lensômetro (f) encontra-se na parte superior da lente. Na figura à direita (1B) subimos a lente, fazendo baixar o feixe luminoso de forma que do lugar (f) passemos para (vl) que corresponde ao que consideramos como o valor dióptrico de longe, isto é, no início da zona de progressão 
que, como veremos mais adiante, existe prisma nos centros ópticos das lentes progressivas, pela própria estrutura da lente que não obedece aos paradigmas da geometria euclidiana ${ }^{(5)}$. O ponto marcado foi tomado como o início da progressão e considerado como centro óptico para longe.

4 - Usamos uma abertura do foco de luz do lensômetro de $5 \mathrm{~mm}$ a partir deste ponto inicial da progressão e descíamos através do corredor progressivo marcando o ponto em que encontrávamos a adição de $0,25 \mathrm{D}$. Ao chegar neste ponto anotávamos a distância que o separava do ponto superior e o valor prismático correspondente (Figura 2A). Obtidos esses valores descíamos gradativa e sistematicamente a cada aumento de $0,25 \mathrm{D}$ na adição, anotando o valor da adição, a distância de cada um desses pontos em relação ao ponto inicial e os valores prismáticos encontrados naquela altura. Fomos avançando acrescentando sempre $0,25 \mathrm{D}$ de adição e fazendo as anotações a cada ponto até chegar à adição de $2 \mathrm{D}$ que arbitramos para o estudo (Figura 2B). A escala usada para as medidas era de $0,25 \mathrm{D}$ e $0,25 \Delta$.

5 - Os valores que correspondem ao início da progressão foram considerados, para efeito de estudo, como os do centro óptico para longe. Foi marcado este ponto. Todos os valores prismáticos e as distâncias em milímetro para as adições $+0,25 \mathrm{D},+0,50 \mathrm{D},+0,75 \mathrm{D}, \ldots,+1,75 \mathrm{D}$ foram anotados e tabulados. Eram os valores intermediários. O maior valor de adição (+2D) foi considerado como o centro óptico de perto.

6 - Este caminho foi tomado usando como unidades 0,25 , tanto dos valores dióptricos como prismáticos. Para os valores prismáticos fazíamos as anotações dos valores tanto horizontais como verticais, e não levamos em conta os valores resultantes diagonais. Isso foi feito para termos um acompanhamento melhor da evolução desses valores.

7 - No final, fizemos os cálculos dos valores limites (correspondentes ao que considerávamos centros ópticos para longe e para perto), por terem valores maiores e possibilitarem um maior rigor das medidas das distâncias entre os centros ópticos, o que permitiram duas medidas: a distância entre os centros ópticos de longe e de perto num plano vertical passando pelo centro óptico de longe (linha 1 da Figura 3) e a distância do centro óptico de perto a esse num plano horizontal passando pelo centro óptico de perto (linha 3 da Figura 3). Tendo essas duas medidas era possível calcular o ângulo de rotação do cone que encerrava a zona de progressão.

Utilizamos para um maior rigor da medida lan-

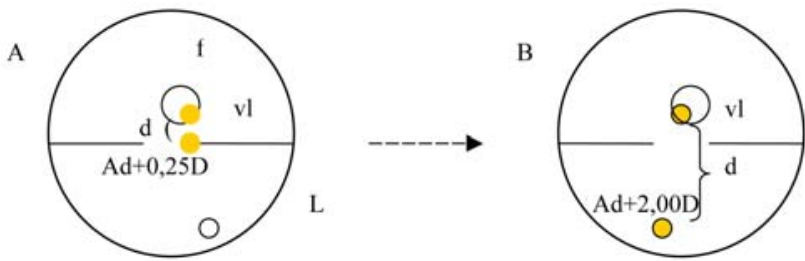

Figura 2: No lado esquerdo (2A), vemos o local da medida onde foi encontrada a adição de $0,25 \mathrm{D}$, que foi marcado, anotado o valor prismático para essa adição e feita a medida da distância que esta adição ficava da parte inicial da progressão (vl) e anotamos o valor da distância (d). Foram feitas medidas sucessivas aumentando em 0,25D a adição sendo repetido o mesmo processo de marcação e de anotação feito para a adição de $0,25 \mathrm{D}$. No lado direito (2B), vemos o exame no local mais superior da adição de 2,00D, sendo anotado, este último como (d). Este ponto era considerado como o centro óptico de perto

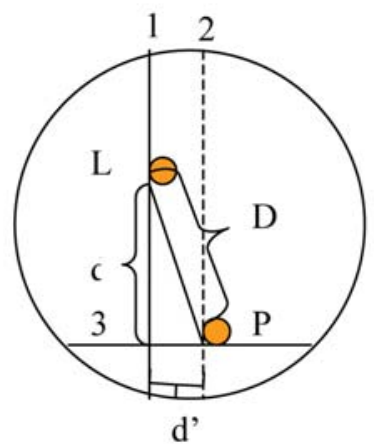

Figura 3: Na representação de uma lente progressiva, vemos o que consideramos centro óptico para longe (L) e centro óptico para perto (P). Tomamos a linha 1 que passa pelo cento óptico de longe e a linha 2 que passa pelo que consideramos centro óptico para perto. A linha 3 tanto tem a projeção do centro óptico de perto no plano do centro óptico de longe (cruzamento das linhas 1 e 3), bem como tem a projeção do centro óptico de longe no plano de centro óptico de perto (cruzamento das linhas 2 e 3). As linhas 1 e 3 permitem determinar os valores $\mathbf{d}$ (distância do centro óptico de longe à projeção do centro óptico de perto) e d' (distância do centro óptico de perto à projeção do centro óptico de longe)

çando mão da unidade de $0,001 \mathrm{D}$, tanto em valores dióptricos como prismáticos, medindo somente o que consideramos como o centro óptico de longe e o de perto, abdicando, devido às dificuldades, das medidas intermediárias por não contarmos com mecanismos sofisticados, sendo usados os meios mecânicos para medidas dos valores das lentes.

\section{Plano de Estudos das lentes}

O estudo se detém inicialmente sobre os prismas que as lentes apresentam nos centros ópticos tanto de longe como de perto. O prisma nos centros ópticos é uma característica fundamental da lente progressiva quando examinada no lensômetro e já reconhecida há quase duas décadas ${ }^{(10)}$.

Para analisar a evolução dos valores da adição, 
tanto da distância em relação a parte superior e dos prismas, usamos o conceito de que "toda função contínua num intervalo fechado é integrável nesse intervalo", usamos essa aplicação da integral de Riemann, da mesma forma que em mecânica se calcula os movimentos uniformemente variados com aceleração inicial $^{(11-12)}$.

Assim usaremos para o cálculo

$$
\int_{a}^{b} f(x) d x=\lim _{0} \sum f(\zeta) \Delta x \quad \zeta=1 / x
$$

Como a extensão da zona de progressão não é a mesma da alteração da curva da superfície progressiva nos propusemos a medi-la. Dessas medidas tivemos que separar as lentes convexas das côncavas e, como havia uma grande variação de extensão, fomos levados a dividir cada tipo de lente em 3 grupos: Grupo I, com a distância entre o início e o fim da progressão inferior a $15 \mathrm{~mm}$; o Grupo II correspondia a extensão tendo de 15 a 20mm e o Grupo III, as lentes com extensão superior a $20 \mathrm{~mm}$. Essa classificação é utilizada ao longo do estudo.
Utilizamos, para melhor compreender como evolui a adição na zona de progressão, a razão incremental. Essa razão incremental é o cálculo do que significa, na extensão da progressão das distâncias, o afastamento do ponto anterior em que se dá o aumento de $0,25 \mathrm{D}$ na adição. Podemos definir a razão incremental como a proporção da distância em que se passa o aumento de uma unidade (no caso, arbitramos como unidade 0,25D) em relação ao total da zona de progressão das lentes progressivas.

A fórmula seria: $\mathrm{I}\left(\mathrm{x}, \mathrm{x}^{1}\right)=\mathrm{d}\left(\mathrm{x}, \mathrm{x}^{1}\right) / \mathrm{zp}$

Em que $\mathrm{I}\left(\mathrm{x}, \mathrm{x}^{1}\right)$ é o intervalo estudado, $\mathrm{d}\left(\mathrm{x}, \mathrm{x}^{1}\right)$ a distância em mm da distância do deslocamento na lente a adição do intervalo escolhido e zp, a extensão total da zona de progressão.

O terceiro ponto que nos deteremos é sobre a inclinação da zona de progressão, para isso partimos dos valores obtidos em medidas pelo método indicado para obter d e d', conforme a Figura 3.

Para estudar a inclinação da zona de progressão usamos duas formas de medida, usando um mesmo método.Assim:

Para o cálculo de $\alpha$ e $\beta$, da Figura 4, sendo $\alpha$, para as lentes convexas, e $\beta$ para as côncavas temos que os

Tabela 1

Comparação do resultado do exame das lentes convexas com Esf+2,00D/Adição +2,00D, medidas com o lensômetro mantendo como unidade $0,25 \mathrm{D}$, usando duas aberturas $\mathrm{e}$ Estabelecendo a diferença entre ambas e o seu percentual

\begin{tabular}{cccccc}
\hline Abertura & \multicolumn{2}{c}{ Prismas para longe } & \multicolumn{2}{c}{ Prismas para perto } & \multicolumn{1}{c}{ Distância longe/perto } \\
\hline $7 \mathrm{~mm}$ & $2,70 \Delta \mathrm{BI}$ & 0 & $2,72 \Delta \mathrm{BS}$ & $0,38 \Delta \mathrm{BT}$ & $18 \mathrm{~mm}$ \\
$5 \mathrm{~mm}$ & $2,13 \Delta \mathrm{BI}$ & 0 & $2,45 \Delta \mathrm{BS}$ & $0,38 \Delta \mathrm{BT}$ & $16,63 \mathrm{~mm}$ \\
Diferença/ percentagem & $\mathbf{0 , 5 7} \Delta(\mathbf{2 6 , 8} \%)$ & $\mathbf{0}$ & $\mathbf{0 , 3 7} \Delta(\mathbf{1 3 , 6} \%)$ & $\mathbf{0}$ & $\mathbf{1 , 3 7 m m ~ ( 8 , 5 \% )}$ \\
\hline
\end{tabular}

Tabela 2

Comparação do resultado do exame das lentes côncavas com Esf-2,00D/Adição +2,00D, medidas com o lensômetro mantendo como unidade $0,25 \mathrm{D}$, usando duas aberturas e estabelecendo a diferença entre ambas e o seu percentual

\begin{tabular}{cccccc}
\hline Abertura & \multicolumn{2}{c}{ Prismas para longe } & \multicolumn{2}{c}{ Prismas para perto } & Distância longe/perto \\
\hline $7 \mathrm{~mm}$ & 0 & $0,04 \Delta \mathrm{BN}$ & $2,34 \Delta \mathrm{BI}$ & $0,18 \Delta \mathrm{BN}$ & $20,27 \mathrm{~mm}$ \\
$5 \mathrm{~mm}$ & $0,25 \Delta \mathrm{BI}$ & 0 & $2,29 \Delta \mathrm{BI}$ & $0,20 \Delta \mathrm{BN}$ & $19,13 \mathrm{~mm}$ \\
Diferença/ percentagem & $\mathbf{0 , 2 5} \Delta \mathbf{B S}$ & $\mathbf{0 , 0 4 B N}$ & $\mathbf{0 , 0 5} \Delta \mathbf{B I}$ & $\mathbf{0 , 0 2 B T}$ & $\mathbf{1 , 1 4 m m}(\mathbf{6} \%)$ \\
& & & $\mathbf{( 2 , 2 \% )}$ & $\mathbf{( 1 0} \%)$ & \\
\hline
\end{tabular}


valores dos ângulos poderão ser conseguidos através da tangente ou da cotangente. Optando pelo cálculo do ângulo da tangente, temos:
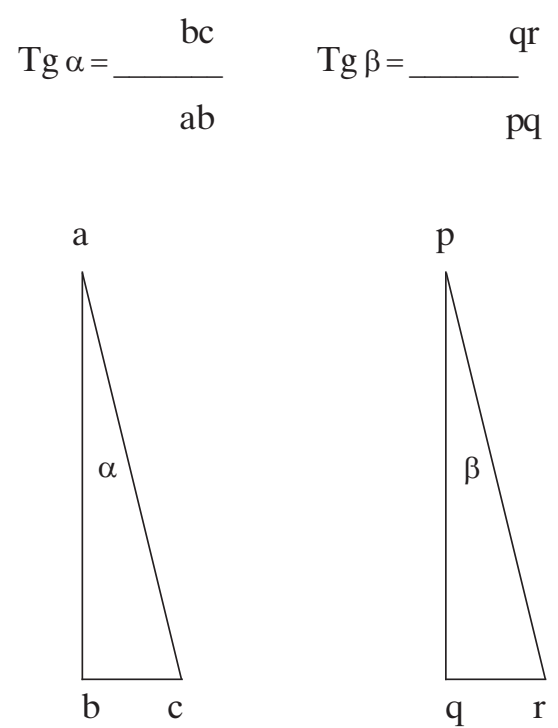

Figura 4: Vemos dois triângulos retângulos abc e pqr, no primeiro nos interessa o ângulo $\alpha$ e no segundo o ângulo $\beta$. Os valores que obtivemos no triângulo abc foram de $\mathbf{a b}$ e bc e eles nos interessam o cálculo do ângulo $\alpha$. Passando para o triângulo pqr temos os valores pq e qr e através deles obtemos o valor do ângulo $\beta$. O triângulo abc corresponde ao achado nas lentes convexas e o pqr nas côncavas

As medidas dos valores acima indicados eram feitas com o compasso, fixadas e transportadas para uma régua que tinham como unidades mínimas em $0,5 \mathrm{~mm}$. Como não dispúnhamos de nada mais rigoroso verificamos que o erro induzido pelo menor rigor não chegaria a $5 \%$ da medida nas linhas o que traria um viés inferior a $0,05^{\circ}$, o que seria insignificante, para os valores que usávamos e para o que queríamos demonstrar. O erro foi calculado previamente e julgado dentro do aceitável para o tipo de medida feita.

\section{Resultados}

Fizemos um estudo comparativo entre os achados com a abertura do fluxo luminoso do lensômetro em $7 \mathrm{~mm}$, que corresponde ao dos lensômetros usados pelos oftalmologistas em consultório e uma abertura menor $(5 \mathrm{~mm})$, que nos permitiu estudar a evolução dos valores dióptricos das lentes progressivas entre longe e perto, e o tomaremos como o "padrão ouro" para o exame das lentes progressivas. Nas Tabelas 1 e 2, vemos a comparação entre as duas medidas.
Tabela 3

A evolução dos prismas ao longo da zona de progressão das lentes progressivas convexas com Correção Esf +2,00D com Adição de 2,00D

\begin{tabular}{lcc}
\hline Grupos & Adição & $\begin{array}{c}\text { Média de valores } \\
\text { prismáticos }\end{array}$ \\
\hline Grupo I & 0D & $\mathbf{1 , 9 4} \Delta$ BI \\
& $0,25 \mathrm{D}$ & $1,68 \Delta \mathrm{BI}$ \\
& $0,50 \mathrm{D}$ & $1,12 \Delta \mathrm{BI}$ \\
& $0,75 \mathrm{D}$ & $0,53 \Delta \mathrm{BI}$ \\
& $1,00 \mathrm{D}$ & $0,05 \Delta \mathrm{BI}$ \\
& $1,25 \mathrm{D}$ & $0,62 \Delta \mathrm{BS}$ \\
& $1,50 \mathrm{D}$ & $0,95 \Delta \mathrm{BS}$ \\
& $1,75 \mathrm{D}$ & $1,38 \Delta \mathrm{BS}$ \\
Grupo II & $\mathbf{2 , 0 0 D}$ & $\mathbf{1 , 8 9} \Delta \mathbf{B S}$ \\
& $\mathbf{0 D}$ & $2,25 \Delta \mathrm{BI}$ \\
& $0,25 \mathrm{D}$ & $1,38 \Delta \mathrm{BI}$ \\
& $0,50 \mathrm{D}$ & $0,88 \Delta \mathrm{BI}$ \\
& $0,75 \mathrm{D}$ & $0,55 \Delta \mathrm{BI}$ \\
& $1,00 \mathrm{D}$ & $0,19 \Delta \mathrm{BI}$ \\
& $1,25 \mathrm{D}$ & $0,79 \Delta \mathrm{BS}$ \\
& $1,50 \mathrm{D}$ & $1,35 \Delta \mathrm{BS}$ \\
& $1,75 \mathrm{D}$ & $1,83 \Delta \mathrm{BS}$ \\
& $\mathbf{2 , 0 0 D}$ & $\mathbf{2 , 6 1} \Delta \mathbf{B S}$ \\
& $\mathbf{0 D}$ & $\mathbf{2 , 5} \Delta \mathbf{B I}$ \\
& $0,25 \mathrm{D}$ & $1,75 \Delta \mathrm{BI}$ \\
& $0,50 \mathrm{D}$ & $1.13 \Delta \mathrm{BI}$ \\
& $0,75 \mathrm{D}$ & $0,75 \Delta \mathrm{BI}$ \\
& $1,00 \mathrm{D}$ & 0 \\
& $1,25 \mathrm{D}$ & $0,69 \Delta \mathrm{BS}$ \\
$1,50 \mathrm{D}$ & $1,19 \Delta \mathrm{BS}$ \\
& $1,75 \mathrm{D}$ & $2,26 \Delta \mathrm{BS}$ \\
$\mathbf{2 , 0 0 D}$ & $\mathbf{3 , 0 6} \Delta \mathbf{B S}$ \\
\hline
\end{tabular}

\section{A evolução do sistema de prismas}

Nas lentes convexas, encontramos inicialmente prisma de base inferior e que com o aumento da adição ele torna-se com valor prismático cada vez menor até ficar num ponto sem nenhum valor prismático. A partir desse ponto, o prisma torna-se de base superior com valor crescente até alcançar o centro óptico de perto.

As lentes côncavas tiveram na nossa amostra, na quase totalidade, um pequeno prisma de base inferior e o valor desse prisma crescia com o aumento da adição (Gráfico 1).

\section{Comportamento das lentes baseado na extensão da zona de progressão}

Fizemos a análise separando os 3 grupos que des- 
Tabela 4

A evolução dos prismas ao longo da zona de progressão das lentes progressivas côncavas com Correção Esf -2,00D com Adição de 2,00D

\begin{tabular}{ccc}
\hline Grupos & \multicolumn{2}{c}{ Média de valores prismáticos } \\
\hline Grupo I & 0D & $0,50 \Delta \mathrm{BI}$ \\
& $0,25 \mathrm{D}$ & $0,68 \Delta \mathrm{BI}$ \\
& $0,50 \mathrm{D}$ & $1,382 \Delta \mathrm{BI}$ \\
& $0,75 \mathrm{D}$ & $1,50 \Delta \mathrm{BI}$ \\
$1,00 \mathrm{D}$ & $1,88 \Delta \mathrm{BI}$ \\
& $1,25 \mathrm{D}$ & $1,88 \Delta \mathrm{BI}$ \\
& $1,50 \mathrm{D}$ & $2,13 \Delta \mathrm{BI}$ \\
& $1,75 \mathrm{D}$ & $2,13 \Delta \mathrm{BI}$ \\
& $\mathbf{2 , 0 0 D}$ & $2,13 \Delta \mathrm{BI}$ \\
Grupo 2 & $0,25 \mathrm{D}$ & $0,33 \Delta \mathrm{BI}$ \\
& $0,50 \mathrm{D}$ & $0,88 \Delta \mathrm{BI}$ \\
& $0,75 \mathrm{D}$ & $1,38 \Delta \mathrm{BI}$ \\
& $1,00 \mathrm{D}$ & $1,63 \Delta \mathrm{BI}$ \\
& $1,25 \mathrm{D}$ & $1,83 \Delta \mathrm{BI}$ \\
& $1,50 \mathrm{D}$ & $2,00 \Delta \mathrm{BI}$ \\
& $1,75 \mathrm{D}$ & $2,08 \Delta \mathrm{BI}$ \\
& $\mathbf{2 , 0 0 D}$ & $2,17 \Delta \mathrm{BI}$ \\
& 0D & $2,25 \Delta \mathrm{BI}$ \\
& $0,25 \mathrm{D}$ & $0,13 \Delta \mathrm{BI}$ \\
& $0,50 \mathrm{D}$ & $0,94 \Delta \mathrm{BI}$ \\
& $0,75 \mathrm{D}$ & $1.56 \Delta \mathrm{BI}$ \\
& $1,00 \mathrm{D}$ & $1,94 \Delta \mathrm{BI}$ \\
$1,25 \mathrm{D}$ & $2,00 \Delta \mathrm{BI}$ \\
& $1,50 \mathrm{D}$ & $2,25 \Delta \mathrm{BI}$ \\
& $1,75 \mathrm{D}$ & $2,44 \Delta \mathrm{BI}$ \\
$\mathbf{2 , 0 0 D}$ & $2,44 \Delta \mathrm{BI}$ \\
& & $2,44 \Delta \mathrm{BI}$ \\
\hline
\end{tabular}

crevemos acima e utilizamos os valores corrigidos para cilindro diagonal, vemos que os menores valores pertencem ao grupo 1 , onde os valores variam de 1,94 de base inferior e se estende até $1,89 \Delta$ de base superior. Os maiores valores estão no grupo 3 , em que a variação se dá entre 2,50 $\Delta$ de base inferior e 3,06 de base superior (Tabela 3).

Nas lentes côncavas os valores dos prismas se deslocam entre os de base inferior (Tabela 4). Os valores menores de prismas que variam no primeiro grupo entre $0,50 \Delta$ e $2,14 \Delta$ de base inferior. A maior variação pertence ao grupo 3 no qual vai de $0,25 \Delta$ e $2,44 \Delta$ de base inferior.

A transição, isto é o local sem prisma na zona de progressão das lentes convexas está exatamente sobre a
Gráfico 1

\section{Evolução dos valores prismáticos \\ das lentes convexas (Esf+2D/Adição +2D) \\ e côncavas (Esf -2D/Adição +2D)}

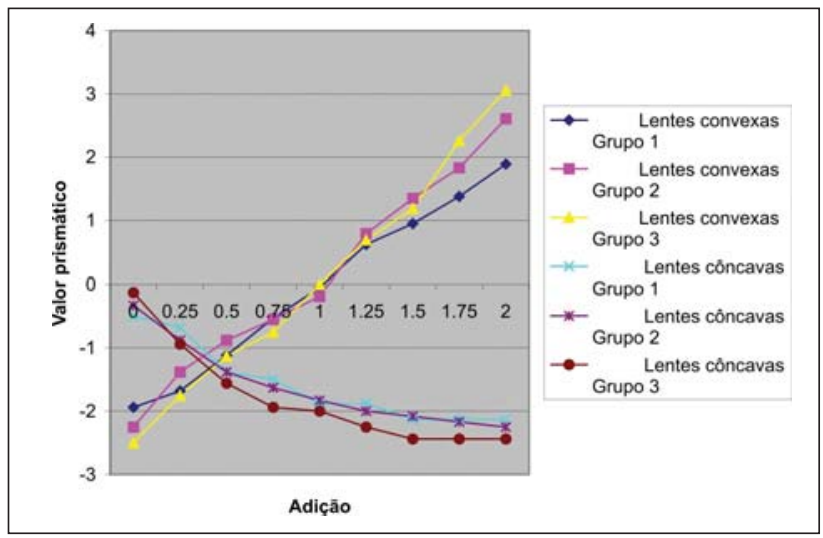

\section{Gráfico 2}

Valores acumulativos das razões incrementais na lente convexa Esf+2D/ Adição +2D

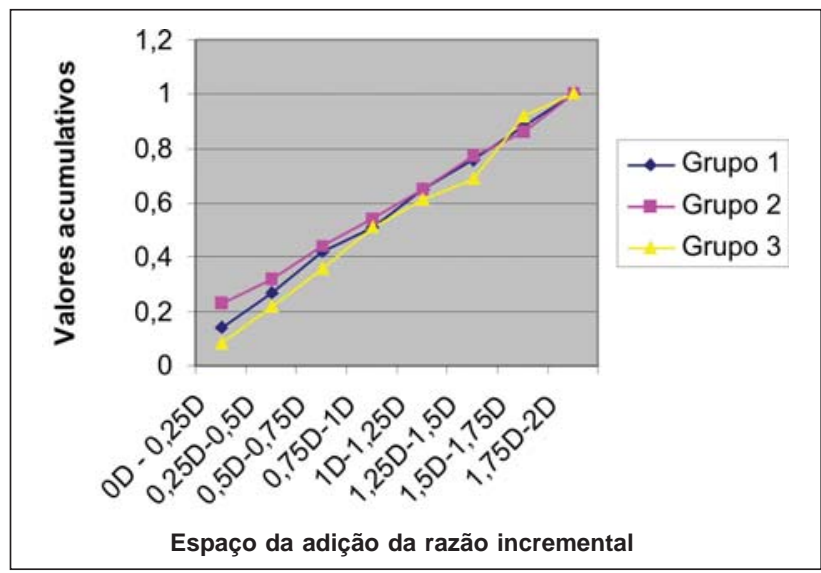

Gráfico 3

Valores acumulativos das razões incrementais na lente côncava Esf-2D/ Adição +2D

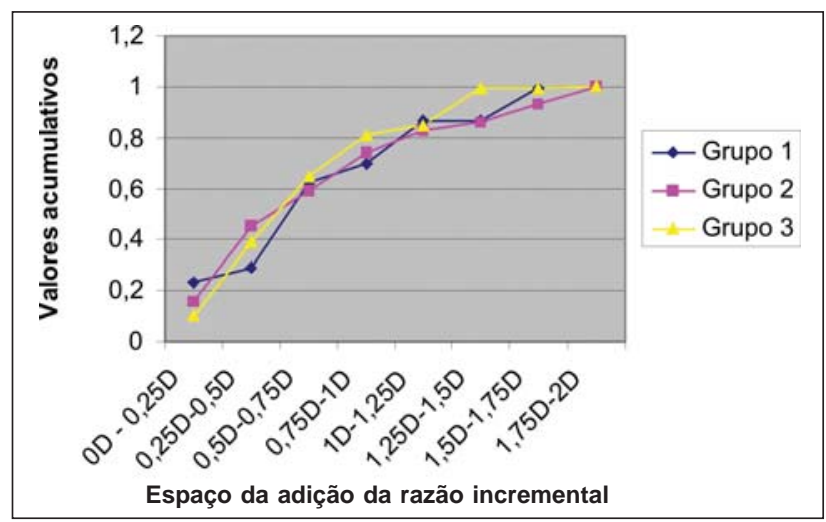


adição de Esf +1,00D, nas lentes do grupo 3. Nos grupos 1 e 2 esse local sem prisma se situa em valores intermediários entre Esf+1,00D e Esf+1,25D (Tabela 3). Vemos que o local sem prisma, nas lentes côncavas, se acha acima do centro óptico e o seu valor poderá ser calculado seguindo a regra de Prentice ${ }^{(13)}$, expressa na fórmula:

$$
\mathrm{d}=\mathrm{c} . \mathrm{f}
$$

Temos que levar em conta as variações das distâncias do ponto inicial da progressão e as adições que tomamos e que correspondem à diferença de $0,25 \mathrm{D}$. Vemos nas Tabelas (5 e 6) para as lentes convexas e côncavas respectivamente.

Fazendo um estudo estatístico, usando o teste de Pearson para as lentes convexas vemos que temos uma forte correlação entre os aumentos incrementais de cada uma das medidas com o aumento dos valores dióptricos das adições crescentes da zona de progressão. Essa forte correlação é encontrada em todos os grupos e com um nível de probabilidade bastante seguro. Nas lentes convexas temos que no Grupo I é de 0,993929; para o grupo 2 é 0,994346 e, para o Grupo 3, 0,989015 com uma probabilidade de $\mathrm{p}<0,01$.

Para as lentes côncavas: no grupo 1 a correlação é de 0,999096, no grupo 2 de 0.990967 e, no grupo 3, 0,995904 com uma probabilidade de $\mathrm{p}<0,01$.

Para compreender melhor a qualidade dos dados de desenvolvimento da progressão podemos ver os Gráficos 2 e 3 .

\section{A análise do ângulo da variação do corredor de progressão}

Como terceiro enfoque da nossa pesquisa, verificamos a angulação que sofre o corredor das lentes progressivas. Vemos que a média das inclinações é maior nas lentes côncavas $\left(6,94^{\circ}\right)$ em relação às convexas $\left(5,87^{\circ}\right)$.

\section{DiscussÃo}

As aberrações astigmáticas têm tomado a preocupação dos estudos das lentes progressivas, deixando de lado as características das suas funções ópticas. As lentes progressivas pela complexidade da geometria da curva, da falta de informações dadas pelo fabricante e a aparente dificuldade de examinar o seu poder dióptrico deixam os oftalmologistas à mercê das informações do marketing das fábricas e do que explicam os ópticos.

Tunnacliffe afirmou que as lentes progressivas de primeira geração tinham uma distribuição simétrica do astigmatismo em redor do corredor da progressão, enquanto que o autor propunha um desenho assimétrico no qual o astigmatismo mudava mais gradualmente dirigido para o lado temporal ${ }^{(14)}$.

Todos os esforços desde o lançamento das lentes progressivas têm sido para solucionar o problema das aberrações astigmáticas. Existem estudos tanto de ordem matemática da superfície das lentes e para exame das lentes com lensômetros que identificam a posição dessas aberrações quantificando os seus valores.

Do ponto de vista matemático, há os que advogam o uso do Polinômio de Zernicke que é um conjunto de equações aplicáveis para uma seqüência de áreas curvas que se deseja aplainar ${ }^{(15)}$, há quem sugira a aplicação da equação de Euler-Lagrange que é uma equação diferencial parcial da elipse, não-linear, de quarta ordem ${ }^{(16)}$; foi também proposto o uso da fórmula de GassBonnet ${ }^{(17)}$ utilizada em aeronáutica para correção das curvas de decolagem e aterrissagem de aeronaves, outros, propõem que sejam usadas alternativas tais como a do B-spline cúbico ${ }^{(18)}$ o que é o uso de dois ou mais parâmetros para facilitar a medida das superficiais ásperas, sendo usada, ainda, para a forma da superfície de veículos automotores. Existe uma proposta muito antiga de Minkwitz baseando-se nos seus estudos sobre superfícies umbilicadas no qual o astigmatismo de segunda ordem (as aberrações astigmáticas) seria determinado junto das vizinhanças da linha vertex estando essa isenta de astigmatismo ${ }^{(19,2)}$. Essa linha vertex seria onde se localizaria a zona de progressão. Muito recentemente, estes estudos foram exumados e dada uma importância incompreensível, embora fossem razoáveis para os conhecimentos de 40 anos atrás. Sheedy et al. mostraram que as relações de Minkowitz podem ser alteradas em algumas regiões do corredor, não existindo uma constante como o descrito embora o conjunto dos componentes possa ter o tratamento da predição do autor ${ }^{(20)}$. Blendowske et al. consideram no estudo da lente progressiva o aspecto da modificação de poder dióptrico local, onde a segunda superfície contribui somente para o valor dióptrico espacialmente constante. $\mathrm{O}$ conceito chave das lentes progressivas seria, para eles, o do chamado umbilicado ou linha vertex ${ }^{(20)}$. Minkwitz imaginou um modelo no qual existia um zona umbilicada onde corria a progressão ficando a correção para longe acima e as para perto abaixo e na lateral as aberrações astigmáticas que decorriam da zona umbilicada ${ }^{(22)}$.

No estágio atual, o problema do desenho da lente é posto como um problema variacional de tentar colocar o valor prescrito sobre a superfície para minimizar o valor total do cilindro ${ }^{(23)}$. 
Tabela 5

Evolução da progressão nos três grupos das lentes convexas e a distribuição da razão incremental das lentes progressivas Esf+2,00D/Adição: Esf+2,00D

\begin{tabular}{|c|c|c|c|c|c|}
\hline Grupos & Adição & $\begin{array}{c}\text { Média da } \\
\text { distância do } \\
\text { centro óptico } \\
\text { para longe }\end{array}$ & $\begin{array}{c}\text { Espaço da } \\
\text { distância de } \\
\text { variação na } \\
\text { adição }\end{array}$ & $\begin{array}{c}\text { Razão incremental } \\
\text { de cada adição } \\
\text { na progressão }\end{array}$ & $\begin{array}{r}\text { Acumulativo } \\
\text { das razões } \\
\text { incrementais }\end{array}$ \\
\hline \multicolumn{6}{|l|}{ Grupo 1} \\
\hline & $0,25 \mathrm{D}$ & 3 & 3 & 0,21 & 0,21 \\
\hline & $0,50 \mathrm{D}$ & 5,5 & 2,5 & 0,17 & 0,38 \\
\hline & $0,75 \mathrm{D}$ & 7,25 & 1,75 & 0,12 & 0,5 \\
\hline & $1,00 \mathrm{D}$ & 9,5 & 2,25 & 0,16 & 0,66 \\
\hline & $1,25 \mathrm{D}$ & 11 & 1,5 & 0,1 & 0,76 \\
\hline & $1,50 \mathrm{D}$ & 12,5 & 1,5 & 0,1 & 0,86 \\
\hline & $1,75 \mathrm{D}$ & 13,25 & 0,75 & 0,05 & 0,91 \\
\hline & $2,00 \mathrm{D}$ & 14,5 & 1,25 & 0,09 & 1 \\
\hline \multicolumn{6}{|l|}{ Grupo 2} \\
\hline & $0,25 \mathrm{D}$ & 3,583 & 3,58 & 0,2 & 0,2 \\
\hline & $0,50 \mathrm{D}$ & 6,333 & 2,75 & 0,15 & 0,35 \\
\hline & $0,75 \mathrm{D}$ & 8 & 1,67 & 0,09 & 0,44 \\
\hline & $1,00 \mathrm{D}$ & 10,083 & 2,08 & 0,11 & 0,55 \\
\hline & $1,25 \mathrm{D}$ & 11,583 & 1,5 & 0,08 & 0,63 \\
\hline & $1,50 \mathrm{D}$ & 13,167 & 1,58 & 0,09 & 0,72 \\
\hline & $1,75 \mathrm{D}$ & 16 & 2,83 & 0,16 & 0,88 \\
\hline & $2,00 \mathrm{D}$ & 18,1667 & 2,16 & 0,12 & 1 \\
\hline \multicolumn{6}{|l|}{ Grupo 3} \\
\hline & $0,25 \mathrm{D}$ & 5,25 & 5,25 & 0,23 & 0,23 \\
\hline & $0,50 \mathrm{D}$ & 7,625 & 2,375 & 0,11 & 0,34 \\
\hline & $0,75 \mathrm{D}$ & 10 & 2,375 & 0,11 & 0,45 \\
\hline & $1,00 \mathrm{D}$ & 12,875 & 2,875 & 0,13 & 0,58 \\
\hline & $1,25 \mathrm{D}$ & 15 & 2,125 & 0,09 & 0,67 \\
\hline & $1,50 \mathrm{D}$ & 17,5 & 2,5 & 0,11 & 0,78 \\
\hline & $1,75 \mathrm{D}$ & 18,125 & 0,625 & 0,03 & 0,81 \\
\hline & $2,00 \mathrm{D}$ & 22,5 & 4,375 & 0,19 & 1 \\
\hline
\end{tabular}

A empresa Essilor assegura o emprego dos polinômios de Zernicke (usando somente 10 dos 36 polinômios), sobre sistemas ópticos otimizados, bem como faz uma modelização matemática de superfícies com funções polinomiais por B-splines ${ }^{(24)}$.

Nos últimos anos, vem sendo feita uma distinção entre progressivas com desenhos rígidos (hard) e flexíveis (soft), nos quais as primeiras tinham uma área de progressão larga e aberração astigmática muito acentuada, as outras com área de progressão mais estreita, astigmatismo das áreas laterais menor ${ }^{(25)}$.

A evolução dos lensômetros para exame das lentes progressivas pode ser acompanhada pelas patentes obtidas no Serviço norte-americano de patentes (Quadro 1), ou através de artigos científicos ${ }^{(26)}$. As patentes obtidas, e que tivemos acesso, foram entre 1970 e 1998 em número de 17. Além dos lensômetros comuns são utilizadas alternativas como as frentes de ondas (wavefront) para ter um estudo mais acurado das aberrações astigmáticas ${ }^{(27)}$.

Os estudos atuais se fazem prioritariamente sobre a superfície da lente. Bell utilizando lensômetro mediu a extensão da zona de visão para longe, extensão da zona de visão intermediária, extensão da zona de visão para perto, e a extensão das aberrações, constando a existência de lentes de qualidade superior e de menor qualidade. Concluiu que não havia considerável diferença entre os elementos testados, sendo o desempenho similar em todas as lentes progressivas, no entanto, a variação do tamanho das zonas é considerável entre as lentes de diversas procedências ${ }^{(28)}$. Os números que obtivemos no presente trabalho desmentem essa afirmação, 
Tabela 6

Evolução da progressão nos três grupos das lentes côncavas e a distribuição da razão incremental das lentes progressivas Esf-2,00D/Adição: Esf+2,00D

\begin{tabular}{|c|c|c|c|c|c|}
\hline Grupos & Adição & $\begin{array}{c}\text { Média da } \\
\text { distância do } \\
\text { centro óptico } \\
\text { para longe }\end{array}$ & $\begin{array}{l}\text { Espaço da } \\
\text { distância de } \\
\text { variação na } \\
\text { adição }\end{array}$ & $\begin{array}{l}\text { Razão incremental } \\
\text { de cada adição } \\
\text { na progressão }\end{array}$ & $\begin{array}{c}\text { Acumulativo } \\
\text { das razões } \\
\text { incrementais }\end{array}$ \\
\hline \multicolumn{6}{|l|}{ Grupo 1} \\
\hline & $0,25 \mathrm{D}$ & 2 & 2 & 0,14 & 0,14 \\
\hline & $0,50 \mathrm{D}$ & 4 & 2 & 0,14 & 0,28 \\
\hline & $0,75 \mathrm{D}$ & 5,875 & 1,875 & 0,13 & 0,41 \\
\hline & $1,00 \mathrm{D}$ & 7,125 & 1,25 & 0,09 & 0,5 \\
\hline & $1,25 \mathrm{D}$ & 9,25 & 2,125 & 0,15 & 0,65 \\
\hline & $1,50 \mathrm{D}$ & 10,625 & 1,375 & 0,1 & 0,75 \\
\hline & $1,75 \mathrm{D}$ & 12,25 & 1,625 & 0,12 & 0,87 \\
\hline & $2,00 \mathrm{D}$ & 13,875 & 1,625 & $\mathbf{0 , 1 2}$ & 1 \\
\hline \multicolumn{6}{|l|}{ Grupo 2} \\
\hline & $0,25 \mathrm{D}$ & 4,083 & 4,083 & 0,23 & 0,23 \\
\hline & $0,50 \mathrm{D}$ & 5,667 & 1,583 & 0,09 & 0,32 \\
\hline & $0,75 \mathrm{D}$ & 7,750 & 2,083 & 0,12 & 0,44 \\
\hline & $1,00 \mathrm{D}$ & 9,583 & 1,833 & 0,1 & 0,54 \\
\hline & $1,25 \mathrm{D}$ & 11,583 & 2,000 & 0,11 & 0,65 \\
\hline & $1,50 \mathrm{D}$ & 13,417 & 1,833 & 0,1 & 0,75 \\
\hline & $1,75 \mathrm{D}$ & 15,333 & 1,916 & 0,11 & 0,86 \\
\hline & $2,00 \mathrm{D}$ & 17,667 & 2,333 & 0,13 & 1 \\
\hline \multicolumn{6}{|l|}{ Grupo 3} \\
\hline & $0,25 \mathrm{D}$ & 1,75 & 1,75 & 0,08 & 0,08 \\
\hline & $0,50 \mathrm{D}$ & 4,75 & 3 & 0,14 & 0,22 \\
\hline & $0,75 \mathrm{D}$ & 7,75 & 3 & 0,14 & 0,36 \\
\hline & $1,00 \mathrm{D}$ & 11 & 3,25 & 0,15 & 0,51 \\
\hline & $1,25 \mathrm{D}$ & 13,25 & 2,25 & 0,1 & 0,61 \\
\hline & $1,50 \mathrm{D}$ & 15 & 1,75 & 0,08 & 0,69 \\
\hline & $1,75 \mathrm{D}$ & 19,5 & 4,5 & 0,21 & 0,9 \\
\hline & $2,00 \mathrm{D}$ & 21,5 & 2 & 0,1 & 1 \\
\hline
\end{tabular}

desde que trabalhando com um grupo de lentes de procedência de uma mesma empresa as discrepâncias eram tão grandes que permitiram classifica-las em três grupos. Um trabalho que se que pesquisou dados de interesse diretamente clínico, medindo a acuidade visual, foi feito laboratorialmente com o exame de 3 olhos, sendo quantificada em diversas posições do olhar ${ }^{(29)}$.

Para que se possa abordar clinicamente as lentes progressivas é necessário examina-las de forma dirigida para relacionar as alterações de confecção com os sintomas apresentados pelos usuários de lentes progressivas.

Tomamos como tarefa inicial a de observar se o método de exame das lentes descrito na pesquisa era passível de ser usado rotineiramente em consultório. Para isso procuramos comparar o que é possível obter de um lensômetro o mais simples com um fluxo luminoso gran- de com o fluxo luminoso que usamos na pesquisa. As tabelas 1 e 2 mostram que, para o estudo da extensão da zona de progressão tanto nas lentes convexas como nas côncavas, a diferença na extensão não chega a 10\%, e como a zona de progressão raramente ultrapassa $25 \mathrm{~mm}$, vemos que a diferença dificilmente chegará a $2 \mathrm{~mm}$ não comprometendo a avaliação da altura que a armação deve ser adaptada ao rosto para que se dê o máximo de aproveitamento da correção.

A análise da lente montada segue a identificação dos pontos iniciais e finais da lente, que consideramos como os centros ópticos e que permite situar certos pontos e valores que consideramos fundamentais.

Usando a Figura 5 temos nas lentes progressivas, do ponto de vista clínico que observar não somente isoladamente cada ponto mencionado nelas $\left(1,1^{1}, \mathrm{p}, \mathrm{p}^{1}\right)$, mas 


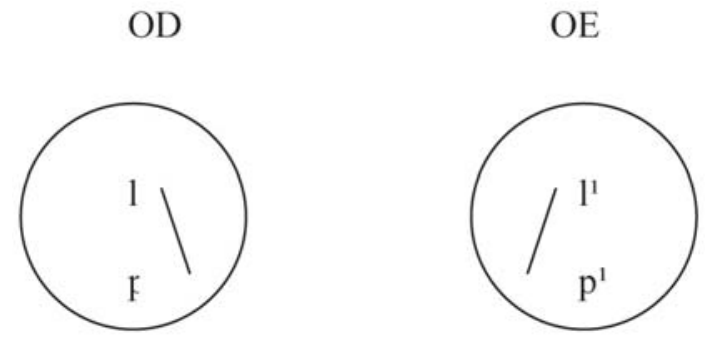

Figura 5: Vemos a representação, como um traço, da zona de progressão em óculos, no qual vemos o início (l, $\left.\mathrm{l}^{1}\right)$ e o seu fim (p,p1), em OD e $\mathrm{OE}$, e que tomamos como fins pedagógicos o centro óptico de longe e o de perto

a relação com a lente do outro lado. Temos que levar em conta não somente a posição do início e do fim da zona de progressão, mas os prismas encontrados em I e l1. Em suma, na verificação das lentes em consultório, temos que verificar: as alturas de $\mathbf{l}$ e $\mathbf{l}^{\mathbf{1}}$, o valor prismático em $\mathbf{l}$ e $\mathbf{l}^{\mathbf{1}}$, a extensão de $\mathbf{l}$ a $\mathbf{p}$ e de $\mathbf{l}^{\mathbf{1}}$ a $\mathbf{p}^{\mathbf{1}}$ e, por fim, as distâncias interpupilares.

Ainda que na seqüência da discussão outros fatores se acrescentaram para acentuar a importância dessas medidas, poderemos chamar à atenção para alguns pontos básicos.

A altura do que consideramos como centro ótico para longe é importante porque, mesmo tendo os centros ópticos o mesmo prisma, o desnível irá determinar um efeito prismático no olhar para longe, podendo ocasionar sintomas de uma heteroforia vertical. Caso estejam na mesma altura, mas tendo prismas diferentes irá também ocasionar um efeito prismático igualmente manifestável por sintomas de heteroforia vertical. A extensão poderá ser mais longa (uma extensão mais curta não traz problemas nem mesmo na visão intermediária) podendo ocasionar dificuldade para longe ou para perto ou para ambas. A distância interpupilar tem importância maior para a visão intermediária, pois, as heteroforias horizontais são melhor toleradas. Uma diferença grande poderá ocasionar sensível perda na visão para as distâncias médias.

Como propusemos o exame em consultório dos valores dióptricos das lentes progressivas usando o foco do lensômetro comum, esse traz a vantagem de determinar o poder dióptrico para longe e para perto numa altura acima do início da zona de progressão e abaixo do fim da progressão, os pontos examinados correspondem a um local de visão utilizável tanto para longe como para perto e, por isso, num local que marcado poderá servir de observação para a adaptação da armação.

Passando a analisar os dados da nossa pesquisa queremos chamar à atenção para o Gráfico 1, em que encontramos uma diferença na variação dos prismas quando se trata de correções com lentes convexas ou côncavas. Estudos mais antigos ${ }^{(4)}$, como os mais recentes ${ }^{(6)}$ e outro no período intermediário ${ }^{(30)}$, além de todos os outros artigos ao longo do tempo, não questionaram a evolução dos prismas na zona de progressão mesmo os admitindo. Vendo de uma maneira evolutiva e defendendo o desenvolvimento das suas lentes, uma empresa coloca que a partir de 1972 foram utilizadas superfícies evolutivas cônicas (elipse, círculo, parábola e hipérbole) e as lentes de $3^{\mathrm{a}}$ geração, os valores através da superfície são os mesmos para a adição de $3,00 \mathrm{D}$ como para a de $1,00 \mathrm{D}{ }^{(31)}$. Como vemos se dá um tratamento optométrico e não se arrisca a emitir uma opinião de interesse clínico.

Encontramos um comportamento diverso do ponto de vista da óptica fisiológica entre os tipos de correção0 convexas e côncavas, o que desmente a visão corrente do comportamento semelhante delas pela análise da superfície das lentes. A evolução dos prismas nas lentes convexas se dá de prisma de base inferior na correção para longe que evolui para um prisma de base superior na correção para perto, formando o que é chamado um conjunto linear de intervalo fechado. Nas lentes côncavas, na nossa amostra a variação se deu com prisma de base inferior que vai crescendo à medida que o valor dióptrico vai da correção para longe no sentido de perto". O Gráfico 1, flagra uma desinformação dada pelas empresas que produzem as lentes progressivas: Elas afirmam que o ponto (.) abaixo do sinal (+) que vem desenhado na lente seria o ponto de neutralização do prisma, ou melhor, o local sem prisma na lente. Ora, olhando no Gráfico 1, vemos que esse local sem prisma encontra-se na zona de progressão das lentes convexas exatamente sobre a adição de Esf $+1,00 \mathrm{D}$, nas lentes do grupo 3 e nos grupos 1 e 2 esse local sem prisma se situa em valores intermediários entre Esf+1,00D e Esf+1,25D, o que corresponderia de 7 a $13 \mathrm{~mm}$ abaixo do início da zona de progressão (Tabela 5). Nas lentes côncavas os valores dos prismas se deslocam entre os de base inferior, ficando a zona sem prisma entre $1,125 \mathrm{~mm}$ e $2,5 \mathrm{~mm}$ acima do

\footnotetext{
"Nas lentes convexas consideramos universal a alteração porque não altera a base do prisma caso se use as lentes ditas equilibradas ou não. Para as côncavas dizemos "(...) na nossa amostra" porque existe uma diferença fundamental nelas devido ao chamado "equilíbrio das lentes". Nessas lentes os prismas deveriam ser de base superior e que iriam decrescendo, no entanto, na amostra, eles são inferiores e de valores crescentes. Esta afirmação se baseia nos dados da formação da imagem e que orientou a escolha da amostra, pois, a adição usada levava de Esf -2,00D até plano (Adição de Esf +2,00D).
} 
Quadro 1

Patentes obtidas no Serviço Norte-Americano de Patentes

\begin{tabular}{llll}
\hline Origem & Número & Autoria & Data \\
\hline USPatent & 3492075 & DAVIS & Janeiro de 1970 \\
USP & 3618271 & MARTIROS & Novembro de 1971 \\
USP & 3991589 & SPITZBERG & Setembro de 1975 \\
USP & 4070115 & HUMPHREY & Janeiro de 1978 \\
USP & 4180325 & HUMPHREY & Dezembro de 1978 \\
USP & 4330203 & OPPENHEIM & Maio de 1982 \\
USP & 4410268 & TAMAKI & Outubro de 1983 \\
USP & 4601575 & TAMAKI & Julho de 1986 \\
USP & 4640596 & HUMPHREY & Maio de 1987 \\
USP & 4730924 & ALLARD & Março de 1988 \\
USP & 5100232 & SMITH et al. & Maio de 1992 \\
USP & 5173739 & KURACHI & Dezembro de 1992 \\
USP & 5175594 & CAMPBELL & Dezembro de 1992 \\
USP & 5303022 & HUMPHREY & Abril de 1994 \\
USP & 5307141 & FUJIEDA & Abril de 1994 \\
USP & 5521700 & KAJINO, KURASHI, & \\
& & NOZAWA e FUJIEDA & Maio de 1996 \\
USP & 5734485 & KAJINO & Março de 1998 \\
\hline
\end{tabular}

início da progressão, que calculamos aplicando aos dados da Tabela 5, a regra de Prentice.

Explicamos o achado nas lentes côncavas pelas informações da empresa ESSILOR, ao explicar o equilíbrio das lentes ${ }^{(32)}$. Pelo o chamado equilíbrio das lentes, as lentes sofrem um afinamento na parte superior que determina o acréscimo ao sistema de prisma das lentes progressivas de prisma de base inferior. As consequiências nas lentes convexas é um aumento do prisma de base inferior para longe e nas lentes côncavas a passagem de prisma de base superior para longe para base inferior. A explicação está na formação de imagem nas lentes progressivas ${ }^{(33)}$.

Tomamos os valores acumulativos das razões incrementais e encontramos uma correlação próxima da perfeição (todas acima de 0,98 ) que é a de valor 1 . Portanto, tivemos uma evolução harmônica das adições intermediárias das lentes da amostra. Mesmo assim, vemos que nas lentes convexas se forma uma linha diagonal ascendente, nas côncavas a linha ascendente forma uma leve concavidade inferior (Gráficos 2 e 3 )

Passando a estudar isoladamente as distâncias entre os valores da adição, levando em conta a razão incremental, verificamos que os espaços não são distribuídos de forma regular havendo comportamentos desiguais nos 3 grupos das lentes convexas, apesar da regularidade da linha ascendente (Gráfico 2). As distâncias entre as unidades de adição nas lentes côncavas apresentam valores relativos decrescentes, mas os valores acumulativos mostram uma concavidade inferior moderada da linha (Gráfico 3). Essas diferenças, embora guardando uma harmonia no geral, são explicadas pelas variadas superfícies geométricas (elipse, círculo, hipérbole e parábola) que constituem as lentes progressivas, mas que dão uma evolução suave da adição.

Por fim, analisaremos os achados relacionados à posição diagonal dos prismas. Nos nossos achados encontramos que nas lentes convexas havia um ângulo de desvio para o lado nasal de $5,86^{\circ}$, enquanto nas lentes côncavas da nossa amostra esse desvio foi de $6,93^{\circ}$.

Os dados mostram uma grande dispersão dos valores. Essa dispersão, justificamos pelo fato de que não controlamos a surfaçagem das lentes, confiando que haveria um erro sistemático do tipo $\hat{a}^{(34)}$, por ser realizado pelas mesmas pessoas utilizando um mesmo instrumental. Para demonstrar que os valores médios não estão fora da realidade tomamos o testemunho de Maitenaz que ao apresentar um requerimento para obter uma patente nos Estados Unidos da América, em 1986, afirma: “(...) a main meridian along at least part of which the curvature of the line varies continuously, and on each side of which the areas of aberration are symetrical to each other, in which lens the main meridian intersects a horizontal plane through the optical center of the lens at a point which, relative to a vertical plane passing through the optical center, is spherically offset toward the nose by between 0,3 to 1,33 millimeters, the plane containing said main meridian is at an angle between 5,5 degrees and 7,5 
degrees to the aforementioned vertical plane, and the main meridian converges toward the nose in the lower part of the lens" ${ }^{\text {(35) }}$. Pode-se ver que os ângulos que encontramos estão dentro da faixa descrita pelo inventor das lentes progressivas atuais.

\section{Conclusão}

Apesar de tomarmos somente duas correções, elas têm uma grande abrangência, pois, tipificam as lentes côncavas e convexas, permitindo conclusões gerais. Achamos importante a divulgação destes dados porque abre uma nova fonte de pesquisas sobre essas lentes e que pode fazer com que os oftalmologistas passem a oferecer novos dados no rumo do conhecimento destas lentes.

Procuramos diferenciar as características da óptica fisiológica das correções das lentes progressivas com lentes côncavas e convexas porque até hoje não foram exploradas visto que os estudos são feitos em lentes planas com diversas adições. O erro básico desses estudos é de que partem do princípio que existe uma superfície progressiva que é o primeiro plano e existem fatores secundários qual seja a do fator dióptrico que determina a correção, enquanto consideramos que existe uma correção em primeiro plano no qual se adiciona uma superfície progressiva. A progressão se ajuntaria às curvaturas básicas e se submeteria a elas embora dando maior complexidade à formação das imagens.

Concluímos que nas lentes devemos examinar a altura do início da progressão para longe, comparar se essa altura está nivelada com a lente do lado oposto; ver se, na mesma altura examinada, os prismas são semelhantes para cada lente dos óculos, medir as distâncias inter-pupilares para longe e para perto e ver em qual posição estão os finais das zonas de progressão. Em outras palavras examinam os 4 pontos examinados nas lentes bifocais, tendo o cuidado de observar o prisma em cada posição superior.

Finalmente queremos reafirmar que não estamos fazendo a crítica da direção dos estudos atuais, pois, o desaparecimento das aberrações astigmáticas é bem vindo, mas essa é uma preocupação para os físicos. Os oftalmologistas devem ficar atentos para a qualidade da confecção das lentes para evitar correções inadequadas e para solucionarem um número elevado de insatisfações com as prescrições de lentes progressivas.

\section{Abstract}

Purpose: This is to study the progressive addition lens $(P A L)$ under the physiological optics' view, to show the lensometer findings and its applicability to daily work. Methods: At first, the aa., explains the ways to examine the $P A L$, and then indicates the physical basis applied to study the prismatic system of the concave and convex lens. It then examines the differences on progression zone extension and explains its diagonal position. Thirteen pairs of lenses from the same manufacturer were examined but every pair had different drawing. The whole (convex and concave lens) had 2 Spherical Dioptries (SD) to distance and an addition of $2 S D$. Results: The convex PAL have to distance correction Inferior Base prism that do lower in progression zone to the point of no prism, and then continues with an increasing Superior Base prism to reach the near correction. The concave PAL has an Inferior Base prism rising constantly to near correction. Both have a mean vertical deviation in the sample of $5,86^{\circ}$ in the convex lenses and $6,93^{\circ}$ in the concave lenses. Conclusion: The aa., based on these findings, shows the most important data to watch in the PAL to minimize the complaints concerning its usage. The studies about PAL highlight that the dioptric values are the background of the progressive zone, and the aa. concludes that the dioptric values are the principal factor and, the progressive zone secondary.

Keywords: Lenses; Visual acuity; Patient satisfation; Comparative study; Models, theoretical; Equipment design

\section{Agradecimentos}

Agradecemos à Empresa SOLA por ter acreditado no nosso empenho em realizar um trabalho de pesquisa sério, e que não lhe trariam benefícios nem financeiros nem de marketing, ao nos fornecer 13 pares de blocos das suas lentes e que nos permitiram realizar o trabalho.

Somos gratos à Óptica "Casa dos Relojoeiros", de Fortaleza, sobretudo na pessoa do Sr. Francisco das Chagas Rocha que encarregou-se de obter a surfaçagem dos blocos de lentes e nos deu acesso ao lensômetro utilizado no estudo, bem como ao proprietário que abriu as suas portas, sem compensação financeira pelo uso do seu laboratório e instalações, e ainda aos funcionários da empresa que tão bem nos acolheram.

\section{ReferênCias}

1. Jalie M. The principles of ophthalmic lenses. 4th ed. London: The Association of British Dispensing Opticians; 1988. p. 256.

2. Reiner J. Auge und Brille. 4th ed. Stuttgart: Ferdinand Enke Verlag; 1987. p. 120-8.

3. Benjamin WJ, Gordon A. Correction with multifocal spectacle lenses. In: Benjamin WJ. Borish's clinical refraction. 2nd ed. St. Louis: Elsevier; 2006. p.1123. 
4. Arbenz JP. Ein neues Mehrstärkenglas. Ophthalmologica. 1975; 170(4): 370-9.

5. Araújo MC, Monte FQ. Lentes progressivas x lentes multifocais: um estudo baseado na geometria analítica do cone. Arq Bras Oftalmol. 2004; 67(3): 393-5.

6. Sheedy J, Hardy RF, Hayes JR. Progressive addition lenses measurements and ratings. Optometry. 2006; 77(1): 23-9.

7. Cho MH, Barnette CB, Aiken B, Shipp M. A clinical study of patient acceptance and satisfaction of Varilux Plus and Varilux Infinity lenses. J Am Optom Assoc. 1991; 62(6): 449-53.

8. Gresset J. Subjective evaluation of a new multi-design progressive lens. J Am Optom Assoc. 1991; 62(9): 691-8.

9. Monte FQ. Erros de confecção das lentes progressivas e suas manifestações clínicas. Arq Bras Oftalmol. 1984; 47(2): 51-6.

10. Atchison DA, Brown B. Prism in pairs of progressive power lenses. Clin Exp Optom. 1989; 72(4):123-33.

11. Ávila G. Calculo I: Funções de uma variável. 6a ed. Rio de Janeiro: Livros Técnicos e Científicos; 1994. p. 27-8.

12. Ávila G. Calculo I: Funções de uma variável. 6a ed. Rio de Janeiro: Livros Técnicos e Científicos; 1994. p. 271-4.

13. Michaels DD. Visual optics and refraction: a clinical approach. 2a ed. St. Louis: Mosby; 1980. p. 47-8.

14. Tunnacliffe AH. Essentials of dispensing. London: The Association of British Dispensing Opticians; 1995. p. 35-72.

15. Les verres progréssifs. Paris: Essilor; s.d. [Cahiers dóptique oculaire, 8].

16. Wang J, Gulliver R, Santosa F. Analysis of a variational approach to progressive lens design. SIAM J Appl Math. 2003; 64(1): 277-96.

17. Curvatures i interpolació en disseny de lents. Report recopilat per Pellicer M. 2003. [Internet]. [cited 2007 Dec 12] Available from: http://www.ma1.upc.edu/recerca/preprints/0304/ 030401pellicer.pdf

18. Otero B, Cela JM, Fontdecaba E. Different surface models for progressives lenses and their effect in parallelization [Internet]. [cited 2008 Abr 29]. Available from: http://www.actapress.com/ PaperInfo.aspx?PaperID $=15560$ \& reason $=500$

19. Minkwitz G. [On the surface astigmatism of a fixed symmetrical aspheric surface]. Opt Acta (Lond). 1963; 10: 223-7. German.
20. Sheedy JE, Campbell C, King-Smith E, Hayes JR. Progressive powered lenses: the Minkwitz theorem. Optom Vis Sci. 2005; 82(10): 916-22.

21. Blendowske R, Villegas EA, Artal P. An analytical model describing aberrations in the progression corridor of progressive addition lenses. Optom Vis Sci. 2006; 83(9): 666-71.

22. Minkwitz G. Bemerkungen über nabelpunktslinien auf flachenstücken. Monatsberichte der deutschen Akademie der Wissenschaften. 1965; 7: 608-10.

23. Loos J, Greiner G, Seidel HP. A variational approach to progressive lens design. Computer Aided Design. 1998, 30(8): 595- 602.

24. Les verres progréssifs. Paris :Essilor ; s.d. p. 19-21. [Cahiers dóptique oculaire, 8].

25. Jalie M. Ophthalmic lenses and dispensing. Oxford: Butterworth-Heinemann; 2001. p. 150-63.

26. Atchison DA, Kris M. Off-axis measurements of a plano distance power progressive addition lens. Ophthalmic Physiol Opt. 1993; 13(3): 322-6.

27. Villegas EA, Artal P. Spatially resolved wavefront aberrations of ophthalmic progressive-power lenses in normal viewing conditions. Optom Vis Sci. 2003; 80(2):106-14.

28. Bell GR. Verifying and evaluating progressive addition lenses in clinical practice. Optometry. 2001; 72(4): 239-46.

20. Villegas EA, Artal P. Visual acuity and optical parameters in progressive-power lenses. Optom Vis Sci. 2006; 83(9):672-81.

30. Wang Q, Yu JC, Chen H. Measurement of progressive addition lenses.Key Engineering Mat. 2008; 364-366: 1048- 53.

31. Les différents types de verres progressifs [Internet]. [cited 2005 Jun 16]. http://research.ac.upc.edu/hpc/Papers/2003/ botero2003aC.pdf

32. Les verres progréssifs. Paris : Essilor; s.d. p. 12. [Cahiers dóptique oculaire, 8].

33. Monte FQ. Modelo de formação de imagens nas lentes progressivas. Arq Bras Oftalmol. 1982; 41(6): 174-80.

34. Schmidt MI, Duncan BB. Epidemiologia clínica e medicina baseada em evidências. In: Rouquayrol MZ, Almeida Filho N. Epidemiologia \& saúde. 6a ed. Rio de Janeiro: MEDSI; 2003. p. 202

35. Maitenaz B. Progressive multifocal ophthalmic lens. United States. US Patent $n^{\circ}$ 4778266. [ Publicado em 18 de Outubro de 1988]. 